\title{
The dwarf nova binary system BV Puppis
}

\author{
A. Bianchini ${ }^{1,3}$, W. Skidmore ${ }^{4,1}$, J. M. Bailey ${ }^{1,2}$, S. Howell ${ }^{5,1}$, and R. Canterna ${ }^{1}$ \\ 1 Dept. of Physics and Astronomy, University of Wyoming, PO Box 3905, Laramie, WY 82071, USA \\ 2 On leave from Campbell County High School, 1000 Camel Dr., Gillette, WY 82716, USA \\ 3 On leave from Dipartimento Di Astronomia, dell'Universitá di Padova, Italy \\ 4 University of St-Andrews, School of Physics and Astronomy, North Haugh, St Andrews, Fife, KY16-9SS, \\ Scotland \\ 5 Astrophysics Group, Planetary Science Institute, 620 N. 6th Ave., Tucson, AZ 85705, USA
}

Received 5 October 2000 / Accepted 4 November 2000

\begin{abstract}
Medium resolution spectra of the dwarf nova BV Pup obtained during an outburst confirm the relatively long orbital period $(P \sim 6.4 \mathrm{~h})$. The emission lines reveal rather structured profiles that are produced in the disc and in the stream. Doppler tomography shows clear bright spot regions in $\mathrm{H} \beta$ and HeII $4686 \AA$ emission line flux. Dynamical solutions for the system parameters are only found if the external radius of the accretion disc is close to the circularisation radius. The white dwarf mass is $1.2 \pm 0.2 M_{\odot}$, the orbital inclination $23^{\circ} \pm 3^{\circ}$.
\end{abstract}

Key words. stars: individual BV Pup - stars: dwarf novae, cataclysmic variables

\section{Introduction}

Dwarf novae are a subclass of cataclysmic variables. A white dwarf accretes matter from a Roche lobe filling companion, usually a lower main sequence star via an accretion disc, quasi-cyclic outbursts occur due to disc instability events (for reviews on this topic see: Frank et al. 1992; Warner 1995). Cataclysmic variables evolve toward shorter orbital periods, angular momentum is lost due to magnetic breaking and/or gravitational radiation. Orbital periods range from about 10 hours to around 1 hour. To test current evolutionary models of cataclysmic variables (Howell et al. 2000), we need to know the system parameters of a large number of these binaries at different orbital periods.

BV Pup is an unusual dwarf nova with small outburst amplitudes ( $m_{v} \sim 15$ in quiescence, $m_{v} \sim 13$ in outburst), short recurrence times ( $\sim 20$ days; Ritter \& Kolb 1998), strong HeII $4686 \AA$ emission (as seen in old novae and nova likes) suggesting a high accretion rate (Szkody et al. 1986, hereafter SHK), and a long orbital period.

EXOSAT observations of BV Pup during outburst show a relatively high hard x-ray flux (SHK), Ginga observations during quiescence fail to detect any flux over background levels (Szkody et al. 1990). However, observations of most dwarf novae show the hard X-ray flux decreases during outburst as the boundary layer becomes optically thick (Cordova \& Mason 1984). The AAVSO light curve

Send offprint requests to: A. Bianchini,

e-mail: bianchini@pd.astro.it of BV Pup shows confused behavior with rapid brightness variations during outbursts. The strong HeII $4686 \AA$ and unusually weak UV line emission during quiescence (SHK) and the lack of HeII $1640 \AA$ emission during outbursts suggest a different stratification of the opacity of the outer disc layers in BV Pup compared to typical dwarf novae (Drew \& Verbunt 1985).

\section{Observations and data reduction}

In this paper we analyze spectroscopic data of BV Pup taken during decline from an outburst. We obtained 38 spectra from 1990 March 27 to April 1 at La Silla using the $1.5 \mathrm{~m}$ ESO telescope equipped with the Boller \& Chivens spectrograph. The spectral range is $4000-5000 \AA$ with a dispersion of $60 \AA / \mathrm{mm}$ and exposure times were 15 $40 \mathrm{~min}$. The spectral resolution is about $1 \AA /$ pixel. All spectra were reduced with the IHAP and IRAF packages. Spectra from nights $1 \& 5$ are shown in Figs. 1 and 2.

Variations of the emission line profiles can be attributed to orbital modulation of the bright spot. The decline of the dwarf nova from an outburst (see next section) is indicated by the disappearance of the high excitation HeII $4686 \AA$ emission line in the spectra of the last two nights. The mean spectra for each night are shown in Fig. 3. Where possible we have measured the radial velocities of both the disc component (mean of the two extreme peaks) and the bright spot component (the central peak) whenever detectable by a multiple Gaussian fit. The estimated errors of the disc and the bright spot 


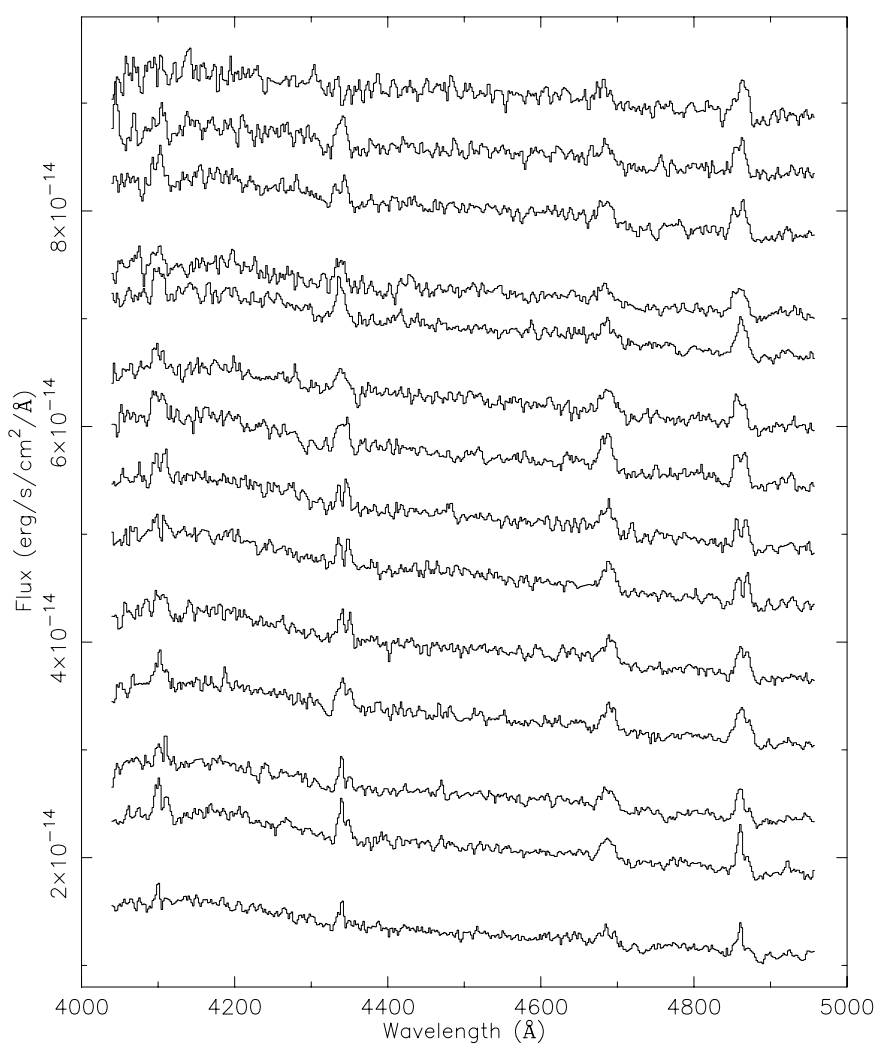

Fig. 1. Fourteen outburst spectra taken on 1990 March 27 (night 1). Time increases up the $y$-axis, an offset of $610^{-15} \mathrm{erg} / \mathrm{s} / \mathrm{cm}^{2} \AA$ has been added to each successive spectrum

radial velocity measurements are $55 \mathrm{~km} \mathrm{~s}^{-1}$ for $\mathrm{H} \beta$ and HeII $4686 \AA$ and $90 \mathrm{~km} \mathrm{~s}^{-1}$ for $\mathrm{H} \gamma$. For this reason the $\mathrm{H} \gamma$ line has not been used to directly derive orbital parameters but has been used when averaging the velocities from different lines. The $\mathrm{S} / \mathrm{N}$ ratio in the continuum near $\mathrm{H} \beta$ was approximately 30 for nights 1 and 2, and 20 for nights 5 and 6 . The $\mathrm{S} / \mathrm{N}$ ratio near $\mathrm{H} \gamma$ was systematically worse due to the poor blue response of the CCD. We estimate the base widths of the emission lines from the mean spectra of nights $1 \& 2$ and nights $5 \& 6$ as the width of Gaussian fits at a 1- $\sigma$ level above the continuum. The journal of the observations with the disc and hot spot radial velocities, the equivalent widths and the fluxes of $\mathrm{H} \beta, \mathrm{H} \gamma$ and $\mathrm{HeII}$, and the fluxes of the continuum at $4400 \AA$ are given in Table 1.

\section{The outburst of BV Pup}

We measured the continuum flux at $4400 \AA$ by averaging the counts in $50 \AA$ wide wavelength bins (centered on $4400 \AA$ ), see last column in Table 1 . The uncertainty on the $4400 \AA$ flux measurements is $17 \%$ (this includes uncertainties in the flux calibration and is greater than the uncertainty due to Poisson noise alone). In Fig. 4, the continuum flux at $4400 \AA$ vs. time shows the decline from an outburst. Assuming a flat continuum between $5000 \AA$ (upper limit of our spectral range) and $5500 \AA$, then $m_{v} \sim 13.8$

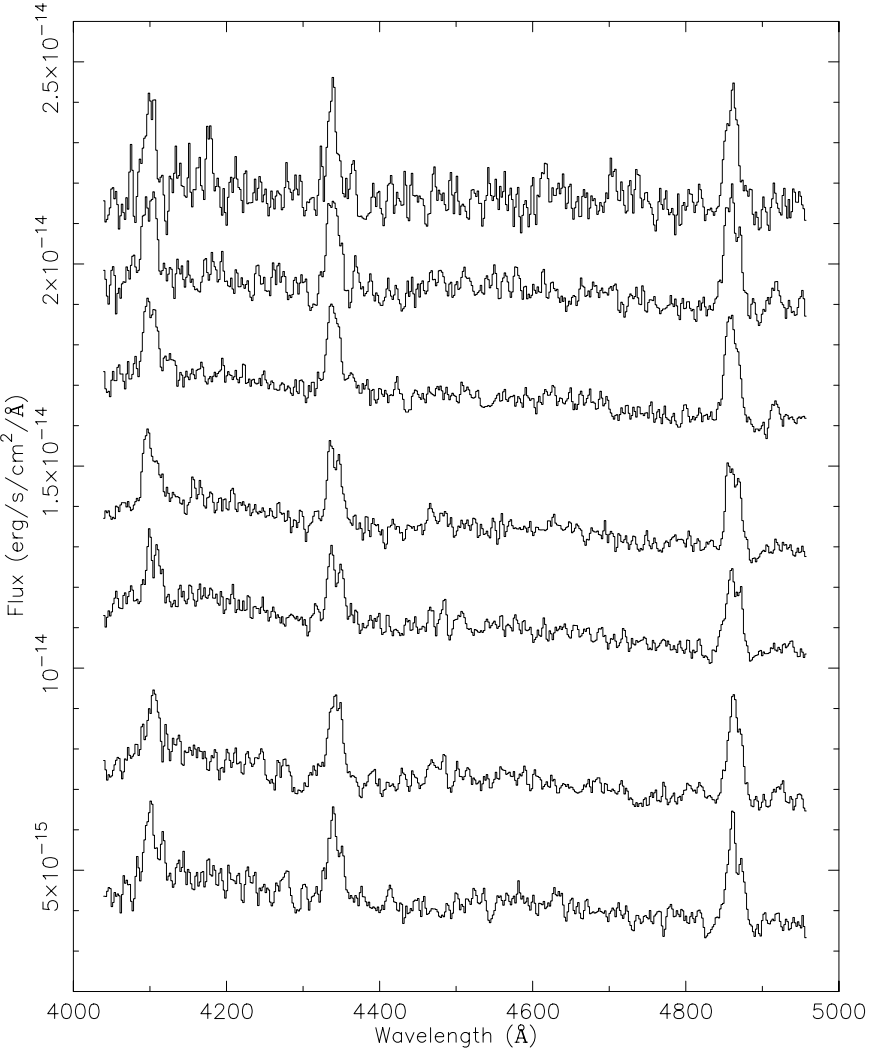

Fig. 2. Seven spectra taken on 1990 March 31 (night 5), BV Pup was nearly 1.4 magnitudes fainter than night 1 . Time increases up the $y$-axis, an offset of $310^{-15} \mathrm{erg} / \mathrm{s} / \mathrm{cm}^{2} \AA$ has been added to each successive spectrum. No HeII $4686 \AA$ lines are visible. The continuum flux has dropped from nights $1 \& 2$ while the line flux has remained constant. The bright spot component is dominating over the disc line emission

on night 1 (HJD 2447977.52), decreasing to $m_{v} \sim 15.2$ on nights $5 \& 6$. AAVSO visual magnitudes for night 1 are $m_{v}=13.6\left(\right.$ JD 2447977.27), $m_{v}=13.4($ JD 2447977.29) and $m_{v}>13.5$ (JD 2447977.83), AAVSO observations performed a few days later state $m_{v}>14$. The previous outburst of BV Pup was recorded by AAVSO approximately 25 days before our first observation, while the outburst following our observations occurred about 20 days later, both outbursts reaching $m_{v} \sim 13$. The epoch of our first spectrum corresponds to the early decline of the outburst.

Figure 3 shows the mean spectrum of BV Pup during nights $1 \& 2$ (outburst) and the mean spectrum in quiescence (average of the spectra from nights $5 \& 6$ ). In outburst the mean spectrum shows a hot continuum, high excitation HeII $4686 \AA$ emission, lower excitation HeI $4471 \AA$ emission and broad absorption features around Balmer lines due to rotationally broadened absorption in the accretion disc (Clark \& Bowyer 1984). No absorption is seen around HeII, this line may be emitted from the hot spot. The line and continuum fluxes in outburst are similar to those observed by SHK. The emission line profiles in Fig. 3 are produced by a combination of the double peaked accretion disc line emission and the single peaked bright 
Table 1. Column 1: observing log. Columns 2-4: disc and bright spot radial velocities, equivalent widths ( $E W \mathrm{~s})$ and fluxes from $\mathrm{H} \beta, \mathrm{H} \gamma$ and HeII line profiles. The uncertainties of the equivalent widths are $\sim 20 \%$. Column 5: the continuum flux at $4400 \AA$

\begin{tabular}{|c|c|c|c|c|c|c|c|c|c|c|c|c|c|}
\hline \multirow[b]{2}{*}{$2447900+$} & \multirow[b]{2}{*}{$\begin{array}{l}\exp \\
\mathrm{s}\end{array}$} & \multicolumn{4}{|c|}{$\mathrm{H} \beta$} & \multicolumn{3}{|c|}{$\mathrm{H} \gamma$} & \multicolumn{4}{|c|}{ HeII } & \multirow{2}{*}{$\begin{array}{c}F_{4400 \AA} \\
10^{-14} \\
\mathrm{erg} / \mathrm{s} / \\
\mathrm{cm}^{2} / \AA\end{array}$} \\
\hline & & $\begin{array}{c}V_{\text {Disc }} \\
\mathrm{km} \mathrm{s}^{-1}\end{array}$ & $\begin{array}{c}V_{\mathrm{HS}} \\
\mathrm{km} \mathrm{s}^{-1}\end{array}$ & $\begin{array}{c}E W \\
\AA\end{array}$ & $\begin{array}{c}F \\
10^{-14} \\
\mathrm{erg} / \mathrm{s} \\
/ \mathrm{cm}^{2}\end{array}$ & $\begin{array}{c}V_{\text {Disc }} \\
\mathrm{km} \mathrm{s}^{-1}\end{array}$ & $\begin{array}{c}V_{\mathrm{HS}} \\
\mathrm{km} \mathrm{s}^{-1}\end{array}$ & $\begin{array}{c}E W \\
\AA\end{array}$ & $\begin{array}{c}V_{\text {Disc }} \\
\mathrm{km} \mathrm{s}^{-1}\end{array}$ & $\begin{array}{c}V_{\mathrm{HS}} \\
\mathrm{km} \mathrm{s}^{-1}\end{array}$ & $\begin{array}{c}E W \\
\AA\end{array}$ & $\begin{array}{c}F \\
10^{-14} \\
\mathrm{erg} / \mathrm{s} \\
/ \mathrm{cm}^{2}\end{array}$ & \\
\hline 77.52041 & 1200 & -8.5 & -45.5 & 4.7 & 4.5 & 72.9 & -86.0 & 1.7 & -6.2 & -63.9 & 3.5 & 4.2 & 1.33 \\
\hline 77.54554 & 1200 & 59.4 & -76.4 & 5.3 & 6.5 & 52.2 & -72.2 & 3.7 & 83.4 & 38.6 & 3.8 & 5.1 & 1.54 \\
\hline 77.56858 & 1200 & 133.4 & -76.4 & 4.8 & 5.4 & 79.8 & -48.4 & 2.4 & -38.2 & -6.2 & 4.0 & 5.0 & 1.41 \\
\hline 77.58687 & 1200 & 195.1 & 65.5 & 5.8 & 7.1 & 93.7 & 24.5 & 3.7 & 102.6 & 128.3 & 3.9 & 5.0 & 1.55 \\
\hline 77.60212 & 1200 & 90.2 & -27.0 & 5.5 & 6.8 & 176.6 & -37.7 & 3.3 & 96.2 & 134.7 & 3.6 & 5.1 & 1.56 \\
\hline 77.61728 & 1200 & 10.0 & -199.8 & 5.5 & 7.0 & 45.3 & -251.9 & 2.5 & 147.5 & 89.8 & 3.7 & 5.0 & 1.67 \\
\hline 77.63241 & 1200 & -14.7 & -193.6 & 4.9 & 6.0 & 100.6 & - & 2.4 & 89.8 & 134.7 & 3.9 & 5.5 & 1.61 \\
\hline 77.64912 & 1200 & -94.9 & -317.1 & 5.0 & 6.3 & -3.1 & -13.5 & 3.8 & 179.5 & 173.1 & 5.2 & 7.1 & 1.60 \\
\hline 77.66448 & 1200 & -70.2 & - & 4.9 & 5.7 & -155.2 & -189.7 & 2.2 & 153.2 & 70.6 & 5.5 & 7.0 & 1.49 \\
\hline 77.67974 & 1200 & -14.7 & -50.7 & 5.1 & 6.2 & -334.9 & -321.0 & 3.2 & 64.2 & 109. & 3.5 & 4.7 & 1.58 \\
\hline 77.69515 & 1200 & -101.3 & -20.9 & 5.7 & 5.9 & -189.7 & -182.8 & 3.8 & -159.9 & -159.9 & 3.9 & 4.2 & 1.30 \\
\hline 77.71190 & 1200 & -33.2 & 96.4 & 6.5 & 7.3 & -169.0 & 162.8 & 3.4 & -44.6 & 38.6 & 3.9 & 4.8 & 1.47 \\
\hline 77.72675 & 1200 & -33.2 & 90.2 & 4.6 & 5.3 & 59.1 & 38.4 & 4.6 & -38.2 & -76.6 & 3.9 & 4.8 & 1.40 \\
\hline 77.74176 & 1200 & -70.2 & 90.2 & 8.0 & 7.9 & - & - & - & 19.4 & -255.9 & 3.8 & 4.5 & 1.33 \\
\hline 77.75628 & 890 & - & - & 8.0: & 7.5: & - & - & 1.8 & -127.8 & -255.9 & 4.0: & 4.5: & 1.25 \\
\hline 78.50706 & 1800 & -45.5 & 53.2 & 6.9 & 5.7 & -44.6 & 114.4 & 3.7 & -313.5 & -377.5 & 3.5 & 3.0 & 1.05 \\
\hline 78.53129 & 1800 & -50.7 & 96.4 & 7.0 & 6.5 & 24.5 & 176.6 & 3.3 & 13.0 & 51.4 & 3.3 & 3.4 & 1.17 \\
\hline 78.56640 & 1800 & -14.7 & -14.7 & 5.8 & 6.4 & 59.1 & -10.0 & 2.9 & 45.0 & -57.4 & 4.0 & 4.1 & 1.40 \\
\hline 78.58820 & 1800 & 40.9 & -64.0 & 7.0 & 8.0 & 24.5 & -58.4 & 4.3 & 96.2 & 6.6 & 3.5 & 4.5 & 1.49 \\
\hline 78.61541 & 1800 & 164.3 & -50.7 & 5.4 & 6.0 & 100.6 & -37.7 & 3.0 & 275.5 & 134.7 & 3.7 & 4.5 & 1.42 \\
\hline 78.63801 & 1800 & 84.1 & -14.7 & 6.5 & 6.9 & 100.6 & -106.8 & 3.4 & 115.5 & 179.5 & 3.5 & 4.1 & 1.39 \\
\hline 78.66072 & 1800 & 96.4 & -50.7 & 5.6 & 5.6 & 93.7 & - & 3.3 & 134.7 & 192.3 & 4.3 & 4.7 & 1.23 \\
\hline 78.68437 & 1800 & 47.0 & -181.3 & 6.0 & 5.4 & 72.9 & - & 2.4 & 166.7 & 121.9 & 4.7 & 4.4 & 1.12 \\
\hline 78.70690 & 1800 & 22.3 & -125.8 & 6.7 & 5.0 & 114.4 & -203.5 & 4.7 & - & 77.0 & 3.9 & 3.4 & 1.01 \\
\hline 79.52119 & 1800 & -57.9 & -14.7 & 5.9 & 3.5 & -272.6 & -348.7 & 2.5 & - & - & 2.0: & 2.8: & 0.69 \\
\hline 81.51476 & 2400 & 77.9 & -27.0 & 18.0 & 6.0 & 100.6 & -93.0 & 7.8 & & & & & 0.41 \\
\hline 81.54564 & 2400 & 96.4 & -2.3 & 15.7 & 5.7 & 79.8 & 72.9 & 7.0 & & & & & 0.41 \\
\hline 81.57558 & 2400 & 96.4 & -113.4 & 13.0 & 5.3 & 10.7 & -286.5 & 6.4 & & & & & 0.50 \\
\hline 81.60466 & 2400 & -2.3 & -45.5 & 13.8 & 5.0 & -16.9 & -196.6 & 8.5 & & & & & 0.44 \\
\hline 81.63682 & 2400 & -39.4 & -88.7 & 14.0 & 5.2 & 52.2 & 100.6 & 6.7 & & & & & 0.47 \\
\hline 81.66631 & 2400 & 28.5 & -82.6 & 18.5 & 6.5 & -148.8 & -37.7 & 8.5 & & & & & 0.42 \\
\hline 81.69069 & 1121 & -70.2 & -8.5 & 19.7 & 6.5 & -72.2 & -99.9 & 10.7 & & & & & 0.36 \\
\hline 82.52738 & 2400 & -50.7 & -64.0 & 22.5 & 8.5 & -65.3 & 10.7 & 11.9 & & & & & 0.43 \\
\hline 82.55885 & 2400 & 47.0 & -39.4 & 22.6 & 9.5 & -65.3 & -72.2 & 12.0 & & & & & 0.51 \\
\hline 82.58849 & 2400 & 47.0 & 53.2 & 22.9 & 9.0 & 107.5 & 3.8 & 15.0 & & & & & 0.46 \\
\hline 82.61922 & 2400 & 108.7 & -33.2 & 17.8 & 8.4 & 59.1 & -10.0 & 12.3 & & & & & 0.54 \\
\hline 82.64854 & 2400 & 40.9 & -144.3 & 18.5 & 7.0 & - & - & 10.5 & & & & & 0.42 \\
\hline 82.67852 & 2400 & 65.5 & -280.0 & 20.5 & 4.8 & -93.0 & -148.2 & 15.0 & & & & & 0.22 \\
\hline
\end{tabular}

spot emission. In quiescence, we see no HeII emission although SHK reported an intensity of about one third that of $\mathrm{H} \beta$.

\section{The spectroscopic period}

The disc radial velocity $V_{\text {Disc }}$ in each spectrum was measured for the $\mathrm{H} \beta, \mathrm{H} \gamma$ \& HeII double peaked emission lines using the mean wavelength of the two peaks, see Cols. 24 in Table 1 . The mean radial velocity for each spectrum was calculated by taking the average of the $V_{\text {Disc }}$ measurements for the $\mathrm{H} \beta, \mathrm{H} \gamma$ \& HeII disc components. Figure 5 shows the power spectra of the mean radial velocity variations of the disc. We interpret the well defined peak corresponding to $P=0.2647 \pm 0.0068 \mathrm{~d}$ as the spectroscopic orbital period of the system. Figure 6 shows the sinusoidal fits to the $\mathrm{H} \beta$, HeII $4686 \AA$ and mean radial velocities of the disc. The systemic velocity $\gamma_{0}$ for BV Pup was calculated using the weighted mean of the $\gamma$ velocities in the fits to the $\mathrm{H} \beta$, HeII and mean radial velocity curves (Table 2), the value of $\gamma_{0}$ for BV Pup is:

$\gamma_{0}=29 \pm 17 \mathrm{~km} \mathrm{~s}^{-1}$

We use the weighted average of the $\mathrm{H} \beta$, HeII and the mean phase offsets (Table 2) to derive the spectroscopic 


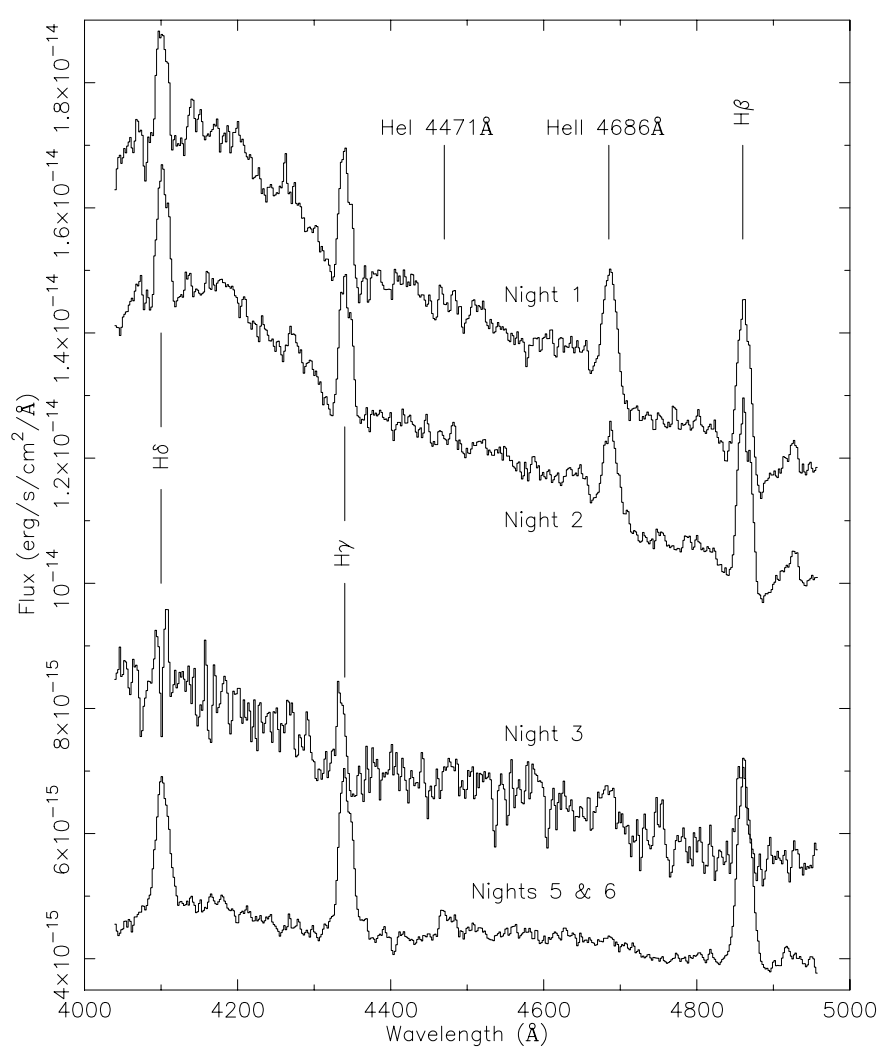

Fig. 3. The mean spectra of nights 1,2 and 3 and the mean spectrum of nights 5 and 6 . The line fluxes are almost the same in all spectra while the continuum drops significantly. HeII $4686 \AA$ is seen only in outburst. In outburst, we see broad absorption features around the Balmer emission lines

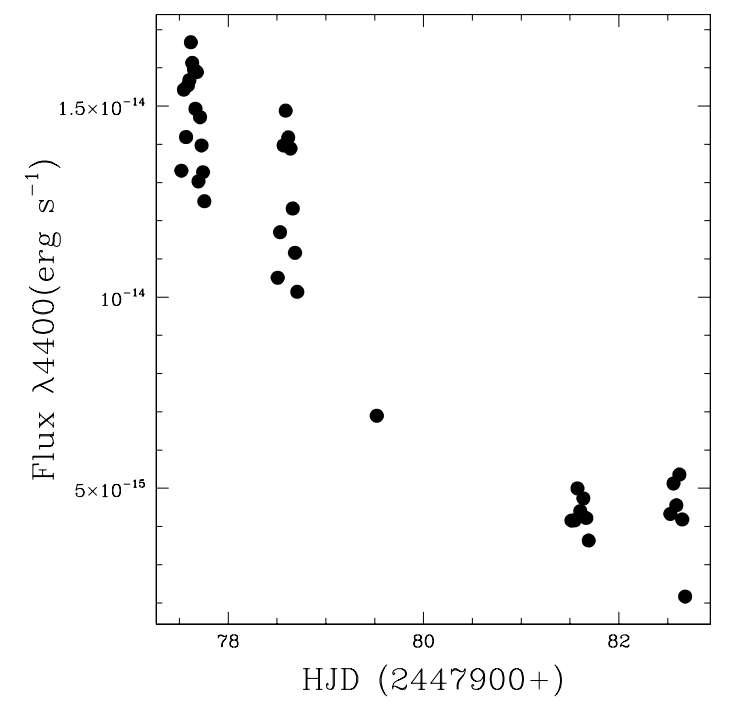

Fig. 4. Observed outburst light curve of BV Pup using the flux of the continuum at $4400 \AA$. The magnitude in outburst is $\sim 13.8$, in quiescence it is $\sim 15.2$

ephemeris for binary phase zero of BV Pup:

$\mathrm{HJD}_{0}=2447977.380 \pm 0.016 P_{\text {orb }}=0.2647 \pm 0.0068 \mathrm{~d}$.

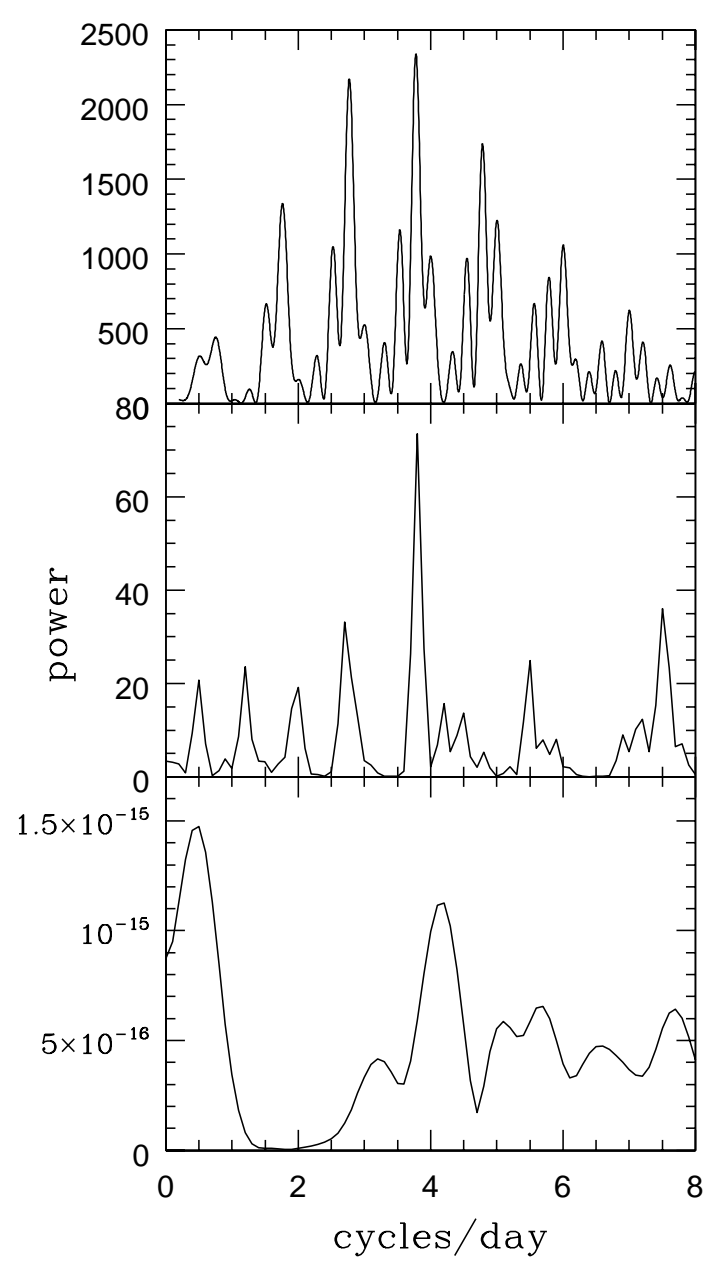

Fig. 5. Top panel: power spectrum of the mean radial velocities derived from averaging $V_{\text {Disc }}$ for $\mathrm{H} \beta, \mathrm{H} \gamma$ \& HeII $4686 \AA$ A. A peak at $3.778 \mathrm{~d}^{-1}(P=0.2647 \pm 0.0068 \mathrm{~d})$ is seen and is confirmed by the power spectrum obtained with the CLEAN program shown in the middle panel. Lower panel: the CLEAN power spectrum of the $4400 \AA$ continuum flux during outburst maximum. The peak at $4.15 \mathrm{~d}^{-1}$ corresponds to $P=$ $0.241 \pm 0.024 \mathrm{~d}$. Thus the peaks are all consistent with each other

Table 2. Results of fits to the radial velocity measurements

\begin{tabular}{ccrc}
\hline Line & $\begin{array}{c}\text { Phase } \\
(\text { Red-blue crossing) }\end{array}$ & $\begin{array}{r}K_{1} \sin i \\
\left(\mathrm{~km} \mathrm{~s}^{-1}\right)\end{array}$ & $\begin{array}{c}\gamma \text { velocity } \\
\left(\mathrm{km} \mathrm{s}^{-1}\right)\end{array}$ \\
\hline $\mathrm{H} \beta$ & $-0.062 \pm 0.030$ & $79 \pm 14$ & $35 \pm 10$ \\
HeII & $0.068 \pm 0.029$ & $111 \pm 18$ & $42 \pm 13$ \\
Mean & $-0.008 \pm 0.025$ & $95 \pm 14$ & $13 \pm 11$ \\
\hline
\end{tabular}

Phasing is wrt $P_{\text {orb }}=0.2647$ days (averaging $V_{\mathrm{R}}$ 's from $\mathrm{H} \beta, \mathrm{H} \gamma$ and $\mathrm{HeII}$ ) and $T_{0}=2447977.380$.

The radial velocities of the hot spot components of $\mathrm{H} \beta$ and HeII, phased according to the new ephemeris do not show pure sinusoidal modulations but sharp minima and rounded maxima (Fig. 7). The $\mathrm{H} \beta$ hot spot component (upper panel) shows a larger scatter and a minimum at $\phi \sim 0.0$. The deep minima and phasing of the $\mathrm{H} \beta$ hot spot radial velocities can be explained using a hot spot 


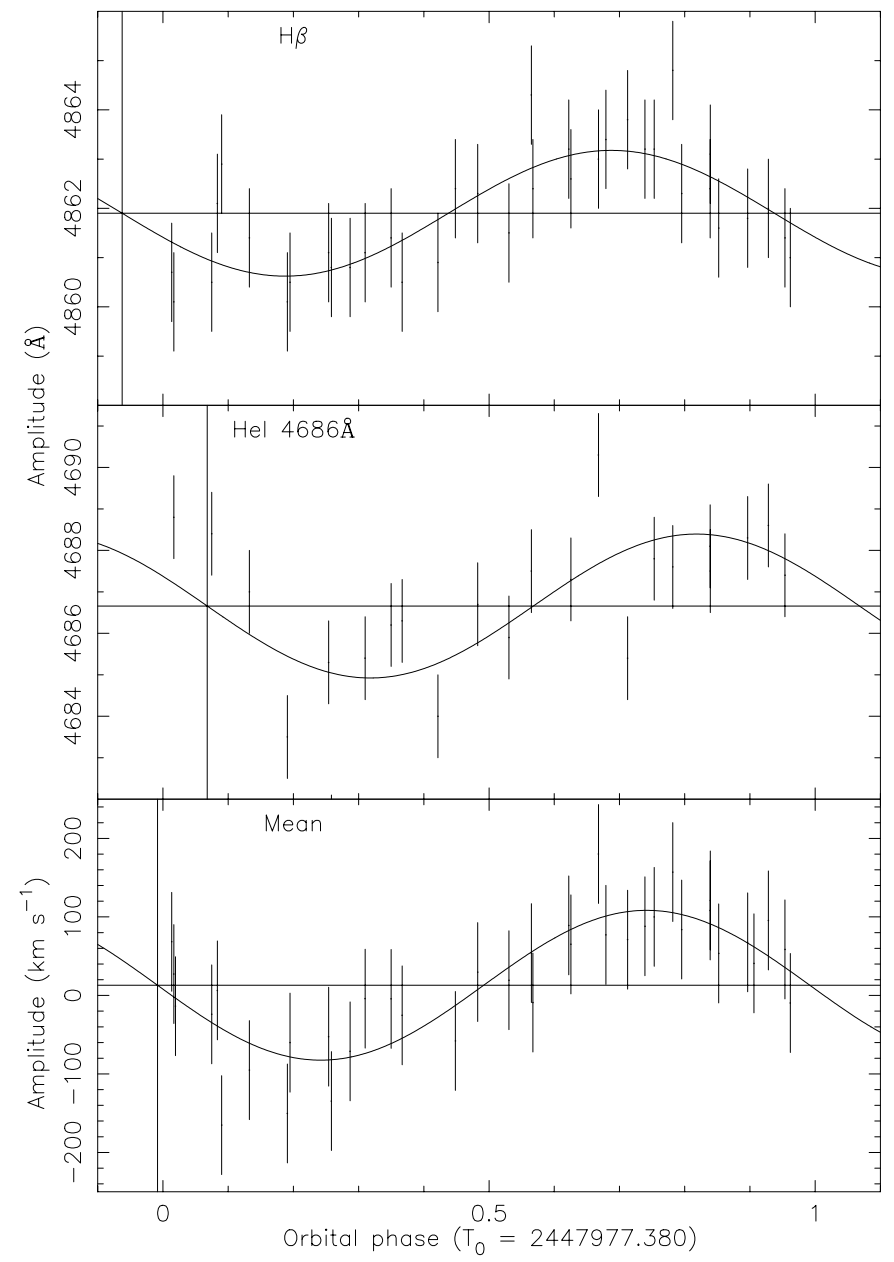

Fig. 6. Top panel: radial velocity curve for the disc component of the $\mathrm{H} \beta$ emission line. Middle panel: radial velocity curve for the disc component of HeII (4686 $\AA$ ). Lower panel: radial velocity curve for the mean of the $\mathrm{H} \beta, \mathrm{H} \gamma$ and HeII values. Phases are referred to HJD $=2447977.380$ derived from the best sinusoidal fits listed in Table 2 . The vertical line marks the position of binary phase 0.0 (corresponding to inferior conjunction of the secondary)

in the usual position, the emitting material moving with Keplerian velocity around the white dwarf, however periodic disappearance of the hot spot line emission around phase zero causes misidentification with the stronger blue shifted peak of the disc line profiles. In the top of Fig. 8, we clearly see the $\mathrm{H} \beta$ bright spot emission except between phases 0.9 to 1.1. The orbital motion of the $\mathrm{H} \beta$ emission wings is clearly seen and is modulated as expected with the red to blue crossing occurring at phase zero. The hot spot component of HeII (lower panel Fig. 7), which is observed only in outburst, has a maximum radial velocity around $\phi \sim 0.9$ and a sharp minimum at $\phi \sim 0.4$, placing the HeII bright spot in the disc slightly down stream from the impacting stream. Figure 8 shows that the HeII hot spot is barely discernible against the broad HeII disc emission. The orbital modulation of the HeII emission line wings can still be seen.

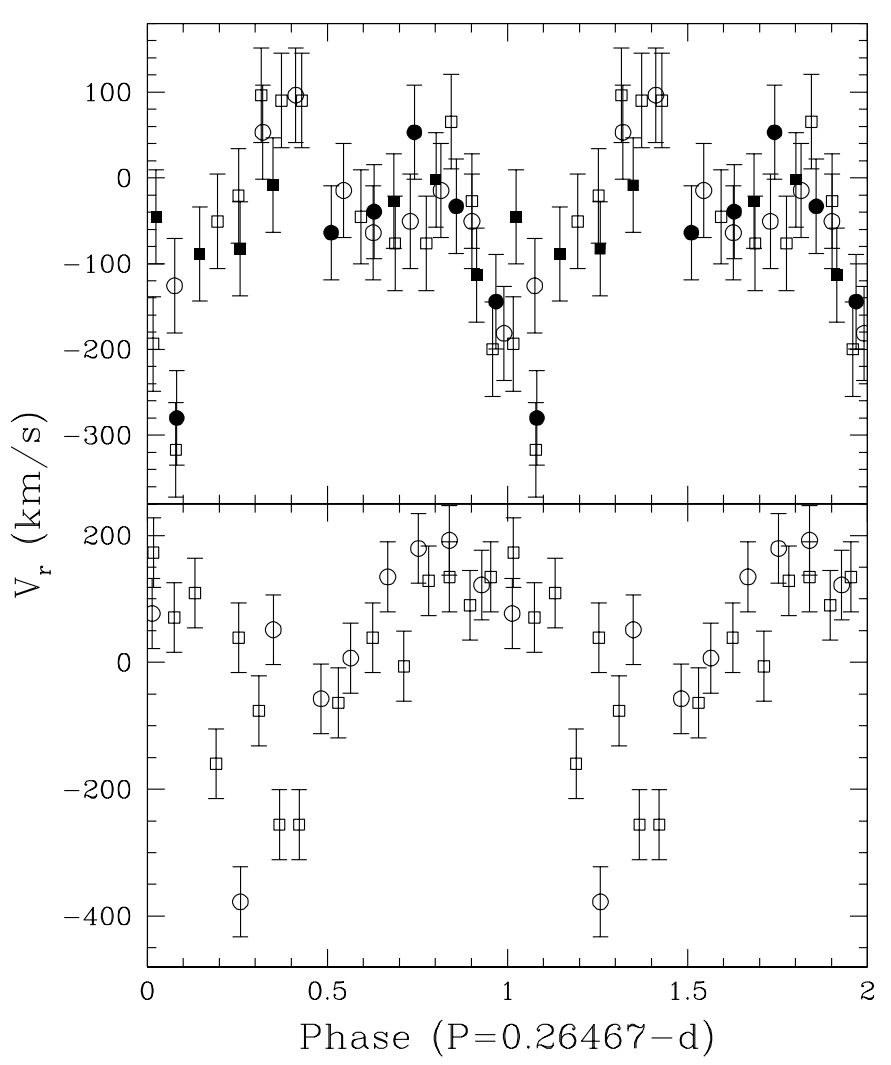

Fig. 7. Top panel: folded radial velocity curve of the $\mathrm{H} \beta$ hot spot component. Open squares indicate night 1 , open circles night 2 , nights $5 \& 6$ filled squares and circles, respectively. $T_{0}=2447977.380$. Bottom panel: folded radial velocity curve of the HeII $4686 \AA$ hot spot component. The maximum at $\phi \sim 0.9$ and the minimum at $\phi \sim 0.4$ suggest that the line emission is produced close to the hot spot region

\section{Equivalent widths and fluxes}

The equivalent widths of the emission lines shown in Fig. 9 display small modulations with the orbital period. The $\mathrm{H} \beta$ equivalent widths are larger by a factor 4 in quiescence than in outburst which is the usual phenomenon for dwarf novae. In our data, the line fluxes in BV Pup do not fade during the decline from outburst. The modulation of the $\mathrm{H} \beta$ equivalent widths is largest in quiescence, with a maximum at $\phi \sim 0.5$, suggesting that the $\mathrm{H} \beta$ flux during outburst is not increased due to irradiation of the secondary star. Little or no modulation is seen in outburst in both $\mathrm{H} \beta$ and HeII. Figure 10 shows a small modulation in the $\mathrm{H} \beta$ and HeII line fluxes with a maximum still around $\phi=0.5$.

In Fig. 10 (bottom panel) we plot the HeII $4686 \AA / \mathrm{H} \beta$ flux ratios vs. orbital period, a saw-tooth trend is seen. The ratios range from 0.5 to 1.1 , in agreement with the observations of SHK and well above the 0.3-0.4 range observed by them when BV Pup was in quiescence. We do not detect any HeII emission in quiescence.

The high intensity of HeII from the bright spot during outburst suggests a high accretion rate. The empirical correlation between the absolute magnitudes, $M_{\mathrm{v}}$, the 

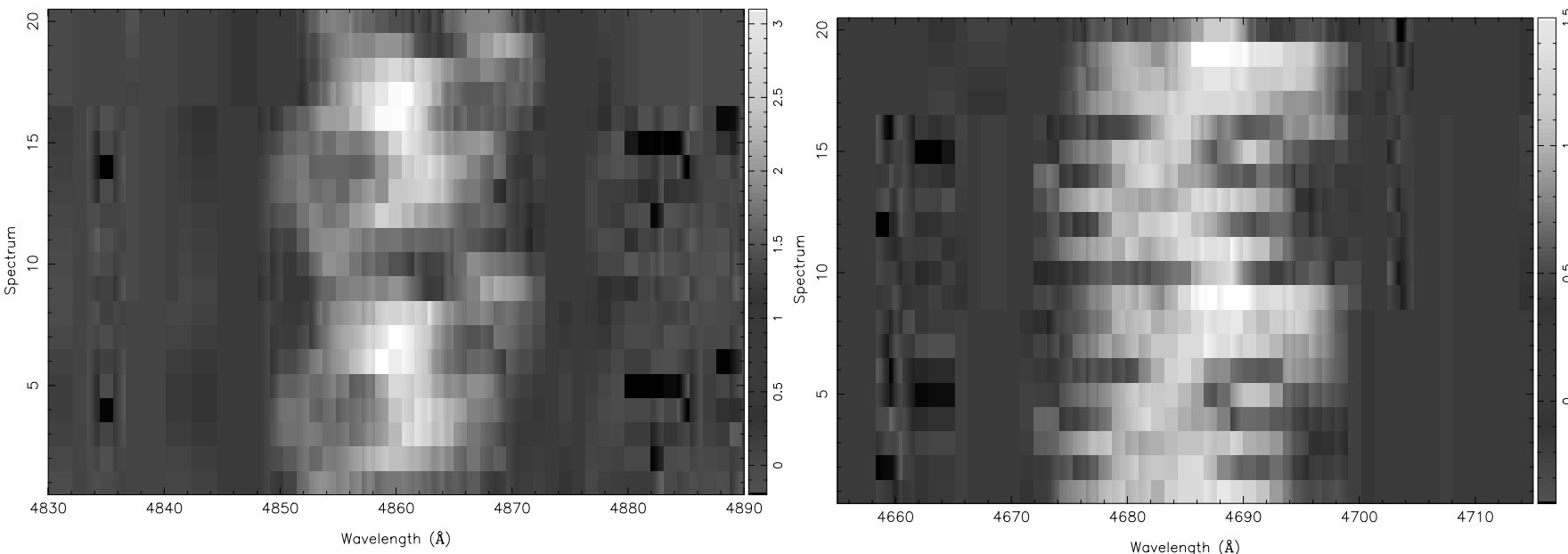

Fig. 8. Left: $\mathrm{H} \beta$ trailed spectrogram. Right: the HeII (4686 $\AA$ ) trailed spectrogram, including data from nights $1 \& 2$. The data were background subtracted as described in Sect. 6, phased according to the spectroscopic ephemeris then binned into 10 equal width phase bins. The 10 phase bins are repeated for clarity. The scale shows the flux in mJy

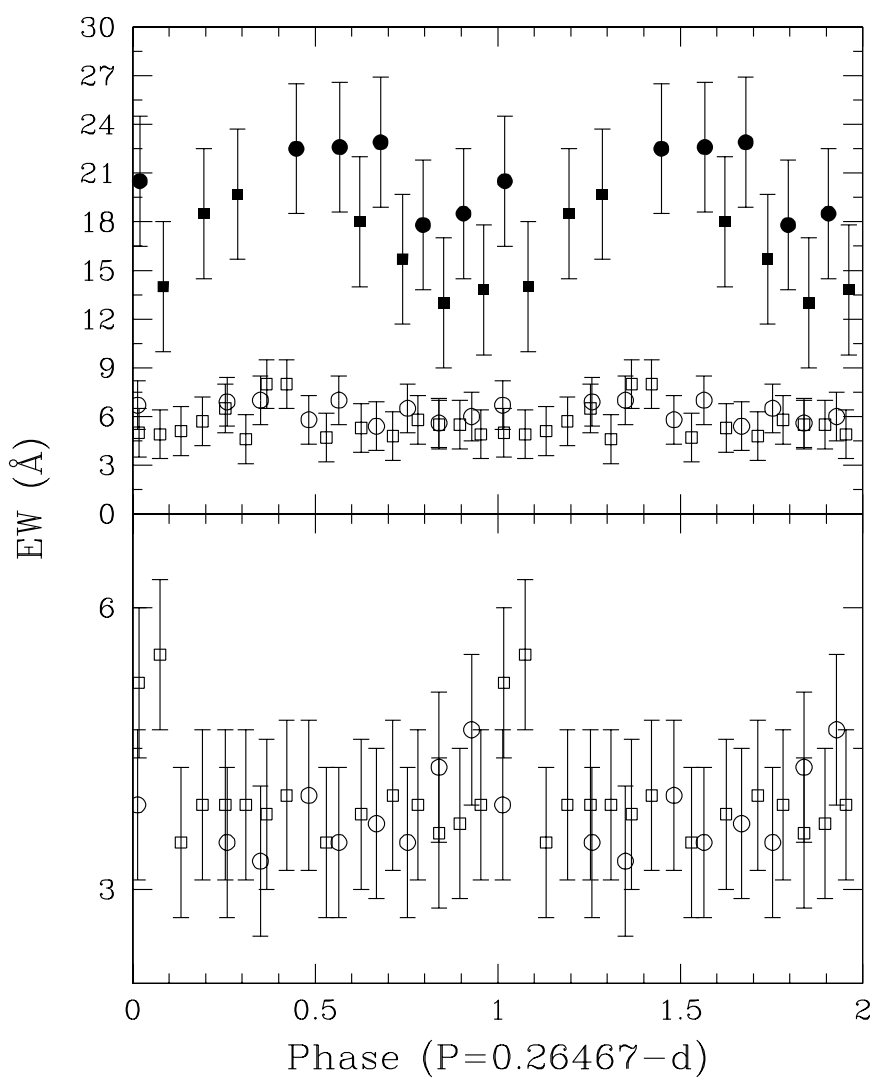

Fig. 9. Top panel: plot of the $E W$ s of $\mathrm{H} \beta$ phased with the new orbital ephemeris. Symbols as in Fig. 7. A modulation with a maximum around phase 0.5 is observed mainly in quiescence. Bottom panel: folded $E W$ s of HeII $4686 \AA$ in outburst; the increase around $\phi=0.0$ is not obvious

outburst recurrence times, $T_{\mathrm{n}}$, and the orbital periods of dwarf novae is (Warner 1987, 1995):

$M_{\mathrm{v}}(\min )=7.11+1.64 \log T_{\mathrm{n}}(d)-0.264 P_{\text {orb }}(h)$.

For BV Pup $\left(T_{\mathrm{n}} \sim 20 \mathrm{~d}\right.$ and $\left.P_{\text {orb }}=0.2647 \mathrm{~d}=6.353 \mathrm{hrs}\right)$ $M_{\mathrm{v}(\min )}=7.58$, the quiescent mass transfer rate is

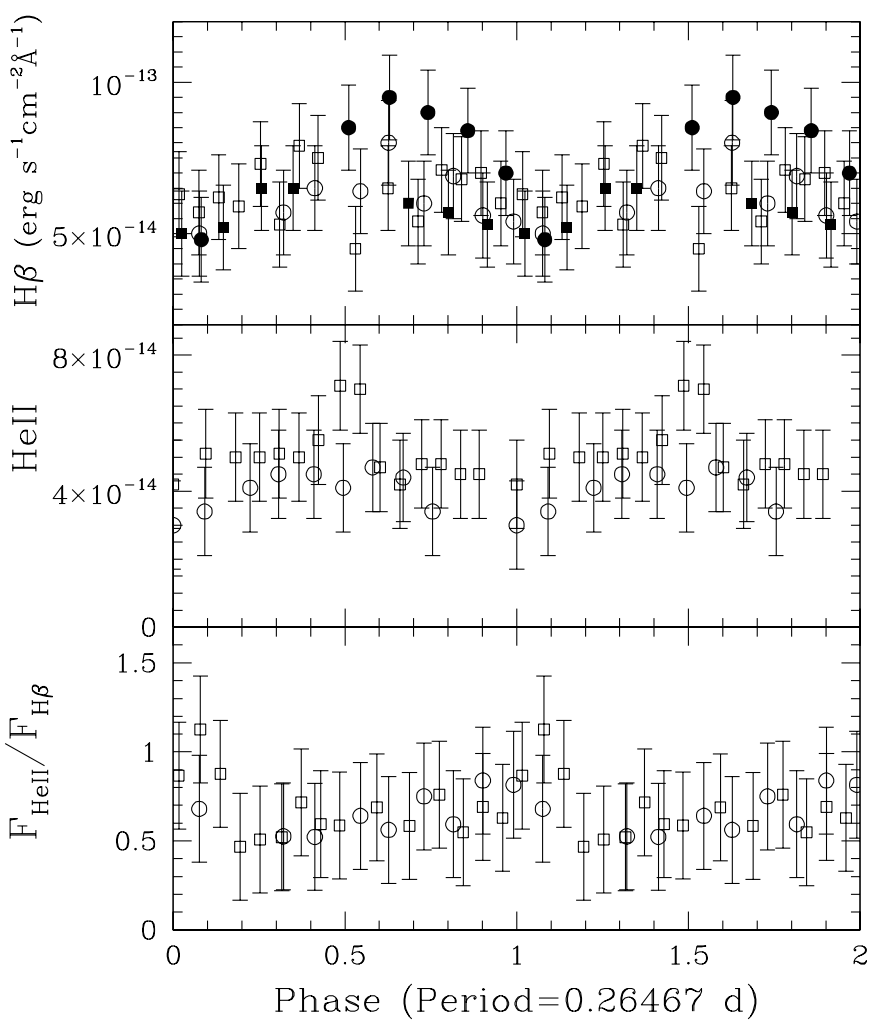

Fig. 10. Top panel: folded $\mathrm{H} \beta$ emission line flux. Symbols as in Fig. 7. As for the $E W \mathrm{~s}$, we see maximum intensity around $\phi=0.6$ that is downstream compared to the standard $\phi=0.8$ bright spot location. Middle panel: folded HeII $4686 \AA$ flux with no significant modulation. Bottom panel: folded HeII $4686 \AA / \mathrm{H} \beta$ flux ratios from nights 1 \& 2

$\sim 610^{15} \mathrm{~g} / \mathrm{s}$ (Smak 1989), this is below the $\sim 310^{16} \mathrm{~g} / \mathrm{s}$ limit theoretically needed to form an optically thick boundary layer that would suppress the hard X-ray flux. Even so, Ginga X-ray observations between $1.5-36 \mathrm{keV}$ of BV Pup in quiescence failed to detect any flux. The $2 \mathrm{mag}$ outburst amplitude gives an outburst mass transfer rate 
through the disc of $810^{16} \mathrm{~g} / \mathrm{s}$. But EXOSAT observations showing a $2-6 \mathrm{keV}$ hard component of $4-510^{-12} \mathrm{ergs} / \mathrm{s}$ and no detection of X-ray flux between $0.5-2 \mathrm{keV}$ during outburst (SHK) suggest a low accretion rate with an optically thin (to X-rays) boundary layer. Clearly the boundary layer in BV Pup behaves in an unexpected way, during quiescence we expect the boundary layer to be optically thin and there to be hard $(\sim 13 \mathrm{keV}) \mathrm{X}$-ray emission, however if it remains optically thick then the hard X-ray emission would be reduced and the lower quiescent accretion rate would mean the boundary layer emission would peak at soft X-ray energies, below the range of the Ginga detectors. Measurements of the soft X-ray flux during quiescence are needed to determine fully the X-ray behaviour of BV Pup.

\section{Doppler tomography}

Doppler tomograms of BV Pup were calculated using Fourier Filtered Back Projection (Horne 1991). As the $\mathrm{H} \beta$ line flux did not change during the duration of the observations, all the data were combined to improve the phase coverage when constructing the $\mathrm{H} \beta$ map. Data from the last two nights were omitted when constructing the He II map. The $\mathrm{H} \beta$ and HeII Doppler maps are shown in Fig. 11. The spectra were phased according to the spectroscopic ephemeris derived in Sect. 4. Before calculating the Doppler maps, the continuum background around the emission lines was subtracted off using a parabola as the underlying continuum was smooth and showed no white dwarf absorption features.

The $\mathrm{H} \beta$ Doppler map (top, Fig. 11) shows an obvious bright spot and an asymmetric ring of disc emission. The variable flux in the $\mathrm{H} \beta$ bright spot (evident in top of Fig. 8) will produce artifacts in the Doppler map and explains the apparent asymmetry in the accretion disc. Due to the low inclination of the system (26 ${ }^{\circ}$, Sect. 7 ), the separation of the bright spot in the Doppler map from the origin at zero velocity is reduced.

The HeII Doppler map (Fig. 11) shows a concentration of emission close to the position of the HeII source suggested in Sect. 4. There is no obvious disc structure in the emission (consistent with Fig. 8) and the bright spot position is at a later phase than the $\mathrm{H} \beta$ bright spot.

We interpret the Doppler maps as showing a $\mathrm{H} \beta$ bright spot with largely Keplerian velocity and a HeII bright spot arising from material that is still on the ballistic trajectory. Both the $\mathrm{H} \beta$ and HeII bright spot fluxes arise from the same physical position.

\section{System parameters}

Using the revised mass-radius/orbital period relationship given by Howell et al. (2000), we derive a secondary mass for BV Pup of $\sim 0.96 M_{\odot}$. Stable mass transfer requires that $q=M_{1} / M_{2} \geq \frac{6}{5}$ (King 1988), giving a lower limit to the primary mass $\sim 1.15 M_{\odot}$.
$H \beta$
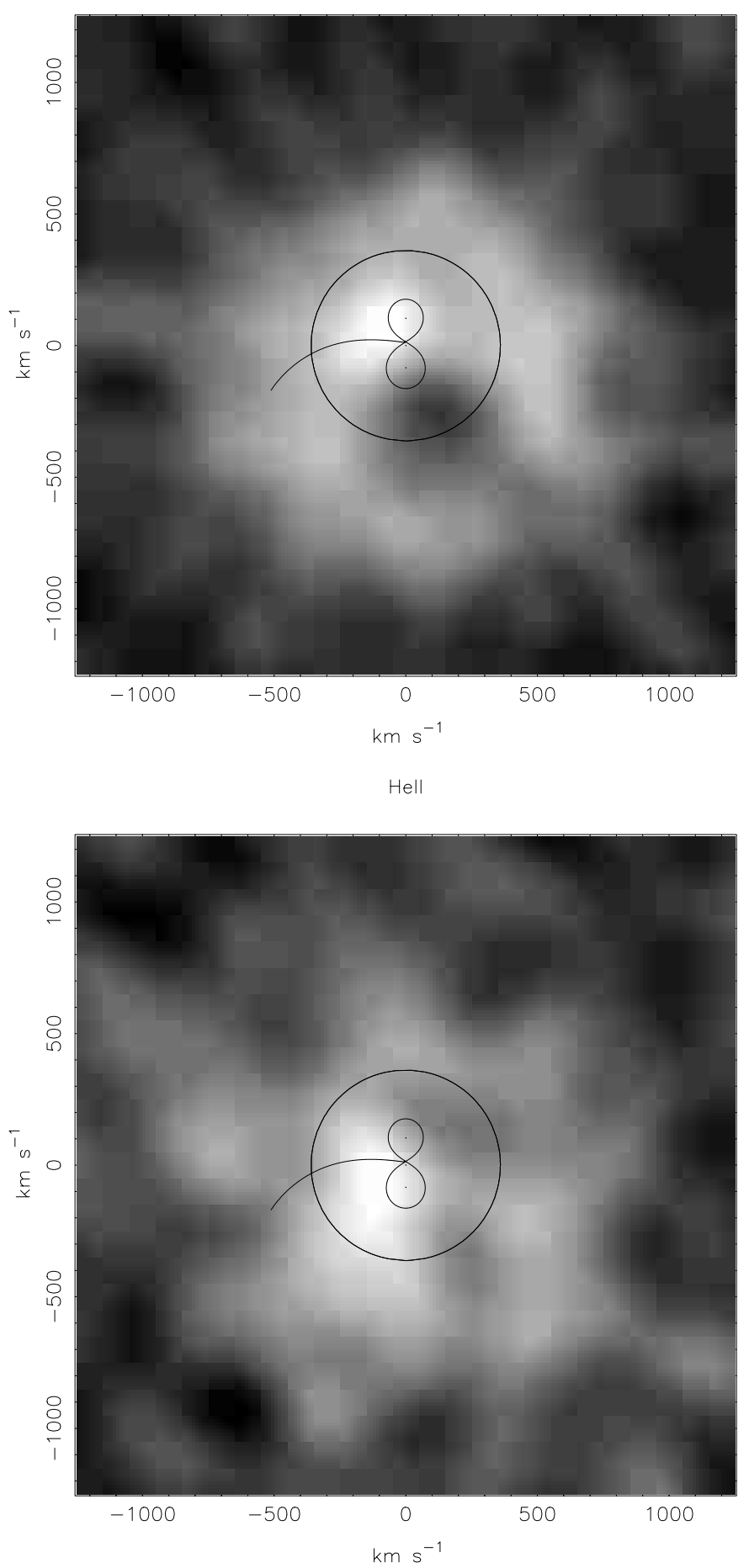

Fig. 11. Top: Doppler map of $\mathrm{H} \beta$. Over-plotted is the system geometry and ballistic stream for the system parameters derived in Sects. 4 and 7, the outer circle represents the keplerian velocity at $\frac{1}{3} R L_{\mathrm{WD}}$. Bottom: doppler map of HeII $4686 \AA$

We can evaluate the primary mass for any orbital inclination of the system using a few assumptions about the system geometry, the orbital velocity of the primary (derived from radial velocity curves) and the Keplerian velocities of the accretion disc material determined from the line profiles. 
The radial velocity curve semi-amplitude, $K_{1}$, represents the orbital velocity of the primary projected along the line of sight. Table 2 lists three values for $K_{1}$. For known values of $K_{1}$ and $M_{2}$ we can use Kepler's third law to derive a relationship between $M_{1}$ and orbital inclination, $i$.

Horne \& Marsh (1986) show that for a simple Keplerian accretion disc the separation of the peaks of the emission line profiles should give the velocity, $V_{\text {out }} \sin (i)$, of the outer rim. However, the line intensity across the disc depends on the radial temperature and density distribution; observations of the low excitation CaII IR triplet in a variety of CVs (Persson 1988) revealed peak separations smaller than those from $\mathrm{HI}$, suggesting that the bulk of the HI emission is produced at a smaller radius. The peak separation may really indicate the velocity of material at the circularisation radius $r_{\text {circ }}$; i.e. the radius where the transferred material accumulates after loosing energy in interactions whilst maintaining the angular momentum about the primary it had at the L1 point (Verbunt \& Rappaport 1988; Hessman \& Hopp 1990). In $\mathrm{BV}$ Pup the circularisation radius $r_{\text {circ }} \sim 0.2 \mathrm{RL} \mathrm{WD}_{\mathrm{W}}$ assuming $q=M_{1} / M_{2} \sim \frac{6}{5}$ where $\mathrm{RL}_{\mathrm{WD}}$ is the white dwarf Roche lobe radius. The observed velocities from double peaked $\mathrm{H} \beta$ profiles of BV Pup are $V_{\text {out }} \sin (i) \sim 380 \mathrm{~km} \mathrm{~s}^{-1}$ in outburst. For a chosen value of $r_{\text {out }}$ and $V_{\text {out }}$ we can define a second relationship between $M_{1}-i$.

The rotational velocities of the inner disc are indicated by the broad absorption profiles around the hydrogen emission lines shown in the mean outburst spectrum from nights $1 \& 2$, this estimate is uncertain because it is difficult to recognize the continuum. Nevertheless, Gaussian fits of the wings at a 1- $\sigma$ level below the assumed continuum indicate velocities of the order of $V_{\text {in }} \sim 2800 \pm$ $200 \mathrm{~km} \mathrm{~s}^{-1}$. Assuming the largest $V_{\text {in }} \sim 2800 \pm 200 \mathrm{~km} \mathrm{~s}^{-1}$ represents the rotational velocity of the inner accretion disc, we can define a third $M_{1}-i$ relationship.

\subsection{Dynamical solution}

In Fig. 12 we plot the three $M_{1}-i$ relationships derived using $K_{1}=79 \mathrm{~km} \mathrm{~s}^{-1}$ (from $\left.\mathrm{H} \beta\right), V_{\text {in }}=2800 \mathrm{~km} \mathrm{~s}^{-1}$ and $V_{\text {out }}=380 \mathrm{kms}^{-1}$ (outburst values) assuming that the bulk of the disc line emission is coming from the circularisation radius, $r_{\text {out }}=r_{\text {circ }}$. Each correlation is represented by two lines corresponding to the $\pm 1 \sigma$ limits of the velocity. The pairs of lines define a common area centered at $M_{1} \sim 1.2 \pm 0.2 M_{\odot}$ and $i \sim 26.0 \pm 3$. This value for $M_{1}$ is consistent with the lower limit of $M_{1}>1.15 M_{\odot}$ derived at the beginning of Sect. 7 .

As long as we assume $r_{\text {out }}=r_{\text {circ }}$, the three $M_{1}-i$ correlations always define a common area for any combination of the orbital velocities of Table 2 and the Keplerian disc velocities. For any other definition of $r_{\text {out }}$ then no common match of the three $M_{1}-i$ relationships is found.

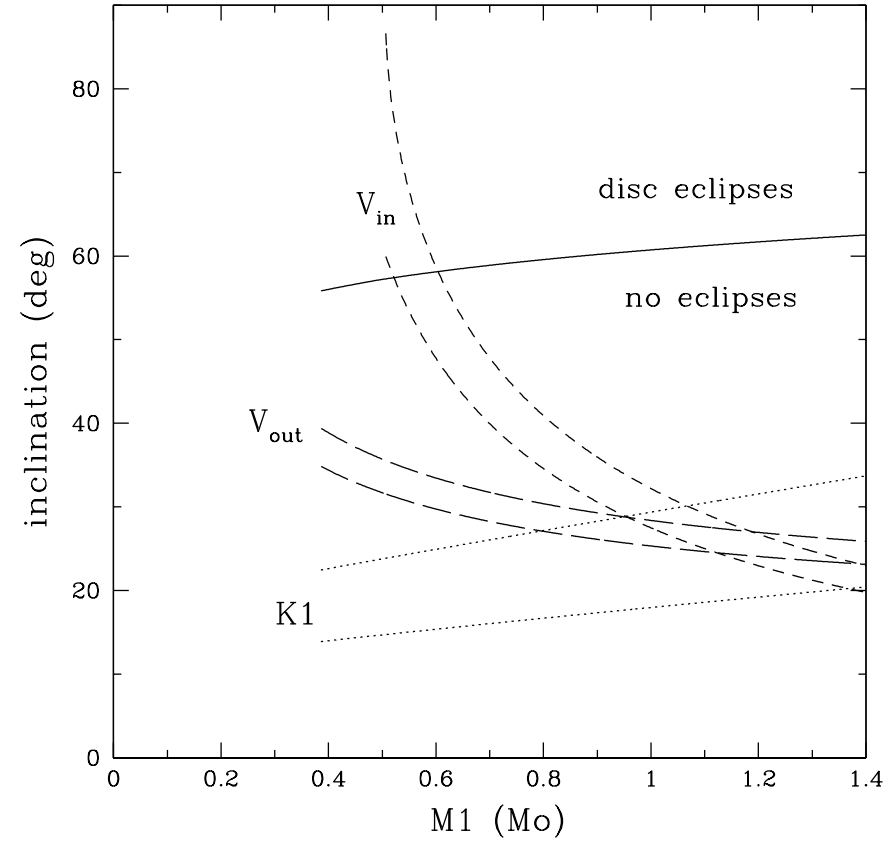

Fig. 12. The relationships between white-dwarf mass and orbital inclination assuming $K_{1}=79 \mathrm{~km} \mathrm{~s}^{-1}, V_{\text {in }}=2800 \mathrm{~km} \mathrm{~s}^{-1}$, $V_{\text {out }}=380 \mathrm{~km} \mathrm{~s}^{-1}$ (outbursting disc) and $r_{\text {out }}=r_{\text {circ }}$. Each correlation is represented by a pair of lines corresponding to the 1- $\sigma$ limits of the input velocity parameter. The best solution is $M_{1} \sim 1.2 \pm 0.2$ and $i \sim 26.0 \pm 3$

\section{Discussion}

We have found a spectroscopic period of 0.2647 days, orbital inclination of $26^{\circ}$ and component masses of $M_{\mathrm{wd}}=1.2 M_{\odot} \& M_{2}=0.96 M_{\odot}$. Some CV systems with orbital periods similar to that in BV Pup show secondary star features in their spectra (Smith \& Dhilllon 1998). Our optical spectra of BV Pup show no evidence for secondary star features. The relatively high accretion rate during quiescence results in a large accretion disc flux that dominates over the secondary star flux. The infrared light curve of Szkody \& Feinswog (1988) shows what may be a double humped modulation as expected for ellipsoidal modulation from the secondary. However the published period for the double humped infrared modulation of $5.4 \mathrm{hrs}$ is longer than the duration of their observations ( $3.3 \mathrm{hrs})$ and therefore it is possible that short timescale structures in the light curve cause the best fit period to be very different to the real orbital period. Further studies of this system should include time resolved infrared spectroscopic observations over several orbital periods in order to properly determine the secondary star characteristics.

\section{Conclusions}

We have confirmed that BV Pup possesses a relatively long orbital period, $P_{\text {orb }}=0.2647 \mathrm{~d}$. If we assume the standard model of a $\mathrm{CV}$ we fail to find self consistent solutions unless we assume $r_{\text {out }}=r_{\text {circ }} \sim 0.12 a \sim 0.2 \mathrm{RL}_{\mathrm{WD}}$.

Doppler tomograms of the $\mathrm{H} \beta$ and HeII $4686 \AA$ lines show clear bright spot regions. The $\mathrm{H} \beta$ bright spot appears 
in the expected position in velocity space for a Keplerian bright spot. The HeII bright spot appears down stream in the disc as deduced from the HeII bright spot radial velocity curve. An asymmetric ring of disc emission is seen in the $\mathrm{H} \beta$ Doppler map, no such structure is seen in the HeII Doppler map. We conclude from the Doppler maps that the majority of the HeII emission emanates from the Bright spot and that the HeII emitting material is still moving along the ballistic trajectory while the $\mathrm{H} \beta$ emitting material is in Keplerian motion about the white dwarf.

Acknowledgements. The $1.5 \mathrm{~m}$ ESO telescope is operated at La Silla by ESO. We thank Tom Marsh for the use of his spectral analysis package MOLLY. SBH acknowledges partial support by NSF grant AST 9732039. One of us (A.B.) wishes to thank the people of the Department of Physics and Astronomy of the University of Wyoming for their friendly hospitality.

\section{References}

Clark, J., \& Bowyer, S. 1984, A\&A, 140, 345

Cordova, F., \& Mason, K. 1984, MNRAS, 206, 879
Drew, J., \& Verbunt, F. 1985, MNRAS, 213, 191

Frank, J., King, A., \& Raine, D. 1992, Accretion Power in Astrophysics (Cambridge University Press)

Hessman, F., \& Hopp, U. 1990, A\&A, 228, 387

Honeycutt, R., Schlegel, E., \& Kaitchuck, R. 1986, ApJ, 302, 388

Horne, K. 1991, Proc. 12th North American workshop on CVs and LMXBs, ed. A. W. Shafter

Horne, K., \& Marsh, T. 1986, MNRAS, 218, 761

Howell, S., Nelson, L., \& Rappaport, S. 2000, ApJ, in press

King, A. 1988, QJRAS, 29, 1

Paczynski, B. 1977, ApJ, 216, 822

Persson, S. 1988, PASP, 100, 710

Ritter, H., \& Kolb, U. 1998, A\&AS, 129, 83

Smith, D., \& Dhillon, V. 1998, MNRAS, 301, 767

Szkody, P., \& Feinswog, L. 1988, ApJ, 334, 442

Szkody, P., Howell, S., \& Kennicutt, R. 1986, PASP, 98, 1151

Szkody, P., Kii, T., \& Osaki, Y. 1990, AJ, 100, 546

Verbunt, F., \& Rappaport, S. 1988, ApJ, 332, 193

Warner, B. 1995, Cataclysmic Variable Stars (Cambridge University Press)

Wood, M., Montgomery, M., \& Simpson, J. 2000, ApJ, 535, L39 